\title{
Television Watching and Mental Health in the General Population of New York City After September 11
}

\author{
Jennifer Ahern \\ Sandro Galea \\ Heidi Resnick \\ David Vlahov
}

\begin{abstract}
SUMMARY. The September 11, 2001 terrorist attacks were watched on television by millions. Using data from a telephone survey of New York City residents in January $2002(N=2001)$, we examined the relations between television watching and probable posttraumatic stress disorder (PTSD) after the attacks. Among those who were directly affected by the attacks or had prior traumatic experiences, watching television was associated with probable PTSD. Experiencing a peri-event panic reaction accounted for some of the association between television watching and probable PTSD. Future research directions are suggested for better understanding the mechanisms behind observed associations between television watching and PTSD.
\end{abstract}

Address correspondence to: Sandro Galea, MD, MPH, DrPH, Center for Urban Epidemiologic Studies, Room 556, New York Academy of Medicine, 1216 Fifth Avenue, New York, NY 10029-5283 USA (E-mail: sgalea@nyam.org).

[Haworth co-indexing entry note]: "Television Watching and Mental Health in the General Population of New York City after September 11.” Ahern, Jennifer et al. Co-published simultaneously in Journal of Aggression, Maltreatment \& Trauma (The Haworth Maltreatment \& Trauma Press, an imprint of The Haworth Press, Inc.) Vol. 9, No. 1/2, 2004; and: The Trauma of Terrorism: Sharing Knowledge and Shared Care, An International Handbook (ed: Yael Danieli, Danny Brom, and Joe Sills) The Haworth Maltreatment \& Trauma Press, an imprint of The Haworth Press, Inc., 2005. Single or multiple copies of this article are available for a fee from The Haworth Document Delivery Service [1-800-HAWORTH, 9:00 a.m. - 5:00 p.m. (EST). E-mail address: docdelivery@ haworthpress.com].

http://www.haworthpress.com/web/JAMT

(C) 2004 by The Haworth Press, Inc. All rights reserved.

Digital Object Identifier: 10.1300/J146v9n01_14 
[Article copies available for a fee from The Haworth Document Delivery Service: 1-800-HAWORTH. E-mail address: <docdelivery@haworthress.com> Website: <http://www. HaworthPress.com> (C) 2004 by The Haworth Press, Inc. All rights reserved.]

KEYWORDS. PTSD, television, panic, trauma, September 11, World Trade Center

The September 11, 2001 terrorist attacks were broadcast live on television and watched by millions as they unfolded. Many networks repeatedly broadcast vivid images of the September 11 attacks, including the collision of the airplanes with the World Trade Center (WTC) towers, people falling from the towers, the towers collapsing, and people running for their lives. One survey conducted soon after the attacks found that $98 \%$ of people in the U.S. watched at least an hour of television coverage on September 11 (Schuster et al., 2001). Considering the wide reach of television into homes across the country, it is important to examine the role television watching may play in shaping psychopathology in the aftermath of a disaster.

Media coverage plays an essential role of communication in disaster situations and also has the potential to affect powerfully those watching (Holloway, Norwood, Fullerton, Engel, \& Ursano, 1997). The relation between television watching and psychopathology in the context of major traumatic events is controversial, with most extant research conducted among children and adolescents (Nader, Pynoos, Fairbanks, Al-Ajeel, \& Al-Asfour, 1993; Pfefferbaum, 2001; Pfefferbaum, Gurwitch et al., 2000; Pfefferbaum, Moore et al., 1999; Pfefferbaum, Nixon et al., 1999; Pfefferbaum, Nixon, Tivis et al., 2001; Pfefferbaum, Nixon, Tucker et al., 1999; Pfefferbaum, Seale et al., 2000).

After September 11, four studies that assessed mental health in the general population of adults demonstrated associations between watching television coverage of the attacks and symptoms of PTSD (Ahern et al., 2002; Schlenger et al., 2002; Schuster et al., 2001; Silver, Holman, McIntosh, Poulin, \& Gil-Rivas, 2002). Our research team conducted a telephone survey of Manhattan residents one month after the September 11 attacks and found associations between watching the image of people falling or jumping from the World Trade Center and probable PTSD (Ahern et al., 2002). This effect was strongest in persons who were directly affected by the attacks (Ahern et al., 2002). Other research found associations between several measures of television watching and symptoms of PTSD in the days and months following the September 11 attacks (Schlenger et al., 2002; Schuster et al., 2001; Silver et al., 2002). 
Current research suggests that the prior experiences of adults watching television may be important factors that shape the association between watching television and psychological distress after a disaster. First, it has been suggested that among those who have been directly affected by a disaster (e.g., those who were injured or lost a close family member in the attacks), watching repeated television images of that disaster may be associated with more substantial negative psychological consequences. This may be due to a personal connection with the event among those who are directly affected and suggests that television watching may exacerbate the effects of a traumatizing experience (Ahern et al., 2002). Second, among people who have experienced other traumatic events in the past, posttraumatic stress symptoms may be exacerbated by watching violent television images (Elliott, 1997; Kinzie, Boehnlein, Riley, \& Sparr, 2002; Kinzie et al., 1998; Long, Chamberlain, \& Vincent, 1994; Moyers, 1996; Pittman, Orr, Forgue, deJong, \& Claiborn, 1987), suggesting that those with prior traumatic experiences may be more affected by television images of a disaster. Third, we have recently shown that those who watch more television after a disaster are more likely to also have experienced a panic attack in the immediate aftermath of the disaster (Ahern, Galea, Resnick, \& Vlahov, in press). Other work has shown that there is a strong relation between experiencing a panic attack soon after a traumatic experience and subsequent posttraumatic stress symptoms (Bryant \& Panasetis, 2001; Deering, Glover, Ready, Eddleman, \& Alarcon, 1996; Falsetti \& Resnick, 1997; Galea et al., 2002), suggesting the possibility that peri-event emotional reactions may play an important role in the relation between television watching and posttraumatic stress symptoms.

Building on this previous research we explore the relations between television watching and probable PTSD in New York City (NYC) after the September 11 terrorist attacks. This exploratory analysis was aimed at generating hypotheses and directions for future research about television watching and its potential relation with posttraumatic stress symptoms among adults after disasters.

\section{METHODS}

\section{Participants}

We conducted a random digit dial telephone survey of NYC residents in January and February of 2002. All persons at least 18 years of age and living in NYC on September 11, 2001 were eligible to participate. We called each telephone number up to 10 times in an effort to contact the residents and inter- 
viewed one person in each household contacted, using a most recent birthday selection method. The interview was approximately 35 minutes in length. All five boroughs of NYC were included in the survey, with an oversample in Manhattan south of 110th Street for comparison with a prior survey. All participants gave informed consent, and the cooperation rate was $63.5 \%$. The cooperation rate is the proportion of persons who were contacted who agreed to participate in the survey, calculated as the completed interviews and screen-outs as a percentage of completed interviews, screen-outs, refusals, and premature terminations. The study protocol was approved by the institutional review board of the New York Academy of Medicine.

\section{Measures}

Television Watching. We assessed television watching by asking how many times respondents had seen the images of "An airplane hitting the World Trade Center," "Buildings collapsing," "People running away from a cloud of smoke or debris," and "People falling or jumping from the towers of the World Trade Center" in the seven days following the attacks. The specific television images and a combined sum of all images were divided into thirds of the number of times people saw them (33.3\% of the population in each group), creating low, medium, and high categories of frequency of watching.

Probable PTSD. We used the National Women's Study (NWS) PTSD module to measure PTSD symptoms. The NWS PTSD module was validated in a field trial against the PTSD module of the Structured Clinical Interview for DSM-III-R (SCID; Spitzer, Williams, Gibbon, \& First, 1992) administered by mental health professionals (Kilpatrick et al., 1998). In the field trial, inter-rater kappa coefficients were 0.85 for the diagnosis of lifetime PTSD and 0.86 for the diagnosis of current PTSD. Comparing the NWS PTSD module to the SCID, the kappa coefficient of the NWS PTSD module with SCID diagnosis of PTSD was 0.77 for lifetime PTSD and 0.71 for current PTSD (Kilpatrick et al., 1998). Instrument sensitivity was $99 \%$ and specificity was $79 \%$ when compared to SCID diagnosis (Kilpatrick et al., 1998; Resnick, Kilpatrick, Dansky, Saunders, \& Best, 1993). The NWS PTSD module assesses the presence of criteria B, C, and D symptoms and determines content for content-specific PTSD symptoms (e.g., content of dreams or nightmares). We assessed probable PTSD since September 11 based on the presence of necessary PTSD criteria $\mathrm{B}, \mathrm{C}$, and $\mathrm{D}$ symptoms during that time period. To measure probable PTSD that was related to the September 11 attacks, all re-experiencing symptoms (criterion B) and all content-specific (e.g., avoidance of thoughts or feelings) avoidance symptoms (criterion $\mathrm{C}$ ) were required to be related to the September 11 attacks. A subset of avoidance symptoms and all the arousal 
symptoms (criterion D; e.g., being easily startled or jumpy) were linked to the attacks by time frame (occurrence since September 11). We use the term "probable PTSD" to reflect the fact that interviews were conducted by lay interviewers using a standard instrument.

We were interested in the role of three factors in shaping the relation between television watching and PTSD.

Being Directly Affected by the Attacks. We assessed whether respondents to our survey were directly affected by the attacks by creating a composite variable of several different potential exposures. We asked respondents about their experiences related to the attacks on September 11, including seeing the WTC attacks in person, being in the WTC complex, being injured in the attacks, having relatives or friends who were killed in the attacks, having possessions lost or damaged in the attacks, being involved in the rescue efforts, and losing a job following the WTC attacks. A respondent experiencing any of the above September 11 experiences was considered "directly affected" by the attacks.

Lifetime Traumatic Events. We assessed lifetime traumatic event experiences by asking if respondents had previously experienced any of the following: a major natural disaster, a serious accident, being attacked with a weapon, being attacked without a weapon but with the intent to seriously injure or kill, forced sexual contact, other serious injury, other situation with potential to seriously injure or kill, seeing someone seriously injured or killed, or any other extraordinarily stressful situation. In this analysis, people who had experienced any of these events were classified as having experienced a previous traumatic event.

Experiencing a Peri-Event Panic Attack. We measured a peri-event panic attack after September 11 by using a modified version of the Diagnostic Interview Schedule measure for panic attack (Centers for Disease Control and Prevention, 1989). Those who reported having four or more symptoms occurring in the "first few hours after" the events of September 11 were considered to have experienced a peri-event panic attack.

\section{Statistical Analyses}

All analyses were conducted in SUDAAN, a statistical software package designed for analysis of cluster-correlated and weighted data (Shah, Barnwell, \& Bieler, 1997), with weighting to account for the probability of selection for interview based on the number of persons and telephone lines in the household as well as the Manhattan oversample.

Relations between the categories of television watching and probable PTSD were assessed in cross-tabulations with two-tailed chi-square tests, and 
relations between continuous measures of television watching and probable PTSD were assessed in bivariate logistic regression models. As the findings revealed the same associations, results for categories of television viewing are presented for ease of interpretation.

We assessed the bivariate associations of the number of times the four television images and a sum of images were viewed with probable PTSD. Next, we assessed these same relations, stratified by whether respondents were directly affected by the attacks. Finally, we assessed the relations between television watching (sum of images) and probable PTSD among combined strata of directly affected and prior traumatic events, and among strata of directly affected with peri-event panic.

\section{RESULTS}

Among survey respondents $(N=2001)$, mean age was 41 years, $53.3 \%$ were female, and the racial/ethnic composition was $40.5 \%$ White, $5.4 \%$ Asian, 25.7\% African American, 25.0\% Hispanic, and 3.3\% of other race/ethnicity. Event exposures were common: $24.6 \%$ saw the attacks on the WTC in person, $12.1 \%$ had a friend or relative killed, $8.8 \%$ were involved in the rescue efforts, and $5.9 \%$ lost a job following the attacks. Overall, $41.2 \%$ were directly affected by the attacks, $69.7 \%$ had a previous traumatic event experience, and $16.7 \%$ experienced a peri-event panic attack.

The image of an airplane hitting the WTC was watched relatively frequently in the 7 days following September $11(M=41.1, M d n=30, S D=35.8)$ as were the images of the buildings collapsing $(M=36.9, M d n=20, S D=$ 34.8 ) and people running from a cloud of smoke $(M=36.7, M d n=21, S D=$ 34.2). The image of people falling or jumping was watched less frequently $(M=$ $11.9, M d n=2, S D=23.8$ ). For all images, the range was 0 to 97 times, with 97 representing 97 or more times in the past 7 days. The sum of all images had a mean of 111.9 times, a median of 70 times and a standard deviation of 111.2.

Respondents who reported watching television images of the attacks on the WTC more frequently in the seven days following September 11 had higher prevalences of probable PTSD, as depicted in Figure 1. Among those who saw an airplane hitting the WTC most frequently, 10.3\% had probable PTSD, while among those who saw it least frequently $4.5 \%$ had probable PTSD $(p=$ 0.003). Similar associations were present for the images of buildings collapsing and people running. For the image of people falling or jumping, there was more probable PTSD among those who saw the image most frequently, but the difference was not statistically significant $(p=0.27)$. As all four images had similar patterns of association with probable PTSD, we created a measure of 
FIGURE 1. Associations between watching television images related to the September 11, 2001 terrorist attacks and probable PTSD, New York City 2002 $(\mathrm{N}=2001)$

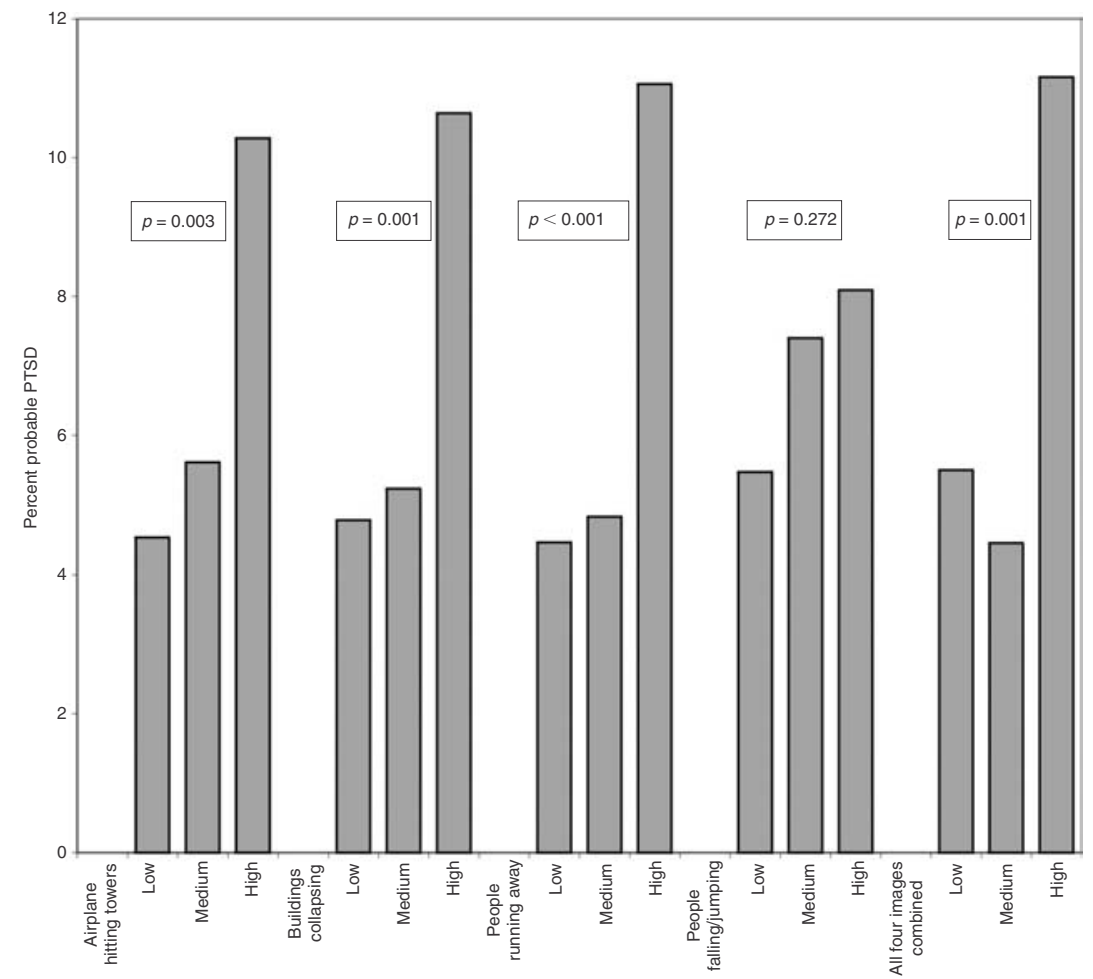

Note: Low, medium and high categories of watching television images correspond to thirds of the number of times respondents reported seeing each image in the seven days following the September 11 terrorist attacks

combined images. For those who saw the most combined images, the prevalence of probable PTSD was $11.2 \%$, and among those who were in the middle and lowest thirds of combined images, the prevalences of probable PTSD were $4.5 \%$ and $5.5 \%$, respectively $(p<0.001$; see Table 1$)$.

The associations between television image watching and probable PTSD, stratified by whether respondents were directly affected, are presented in Table 1 . Among those who were directly affected by the attacks, by low, medium, and high thirds of watching the image of an airplane hitting the WTC, the prevalences of probable PTSD were $7.3 \%, 9.0 \%$, and $14.6 \%$, respectively $(p=0.07)$. The corresponding prevalences for the image of the buildings collapsing were $7.3 \%, 8.3 \%$, and $15.3 \%(p=0.03)$; for the image of people run- 


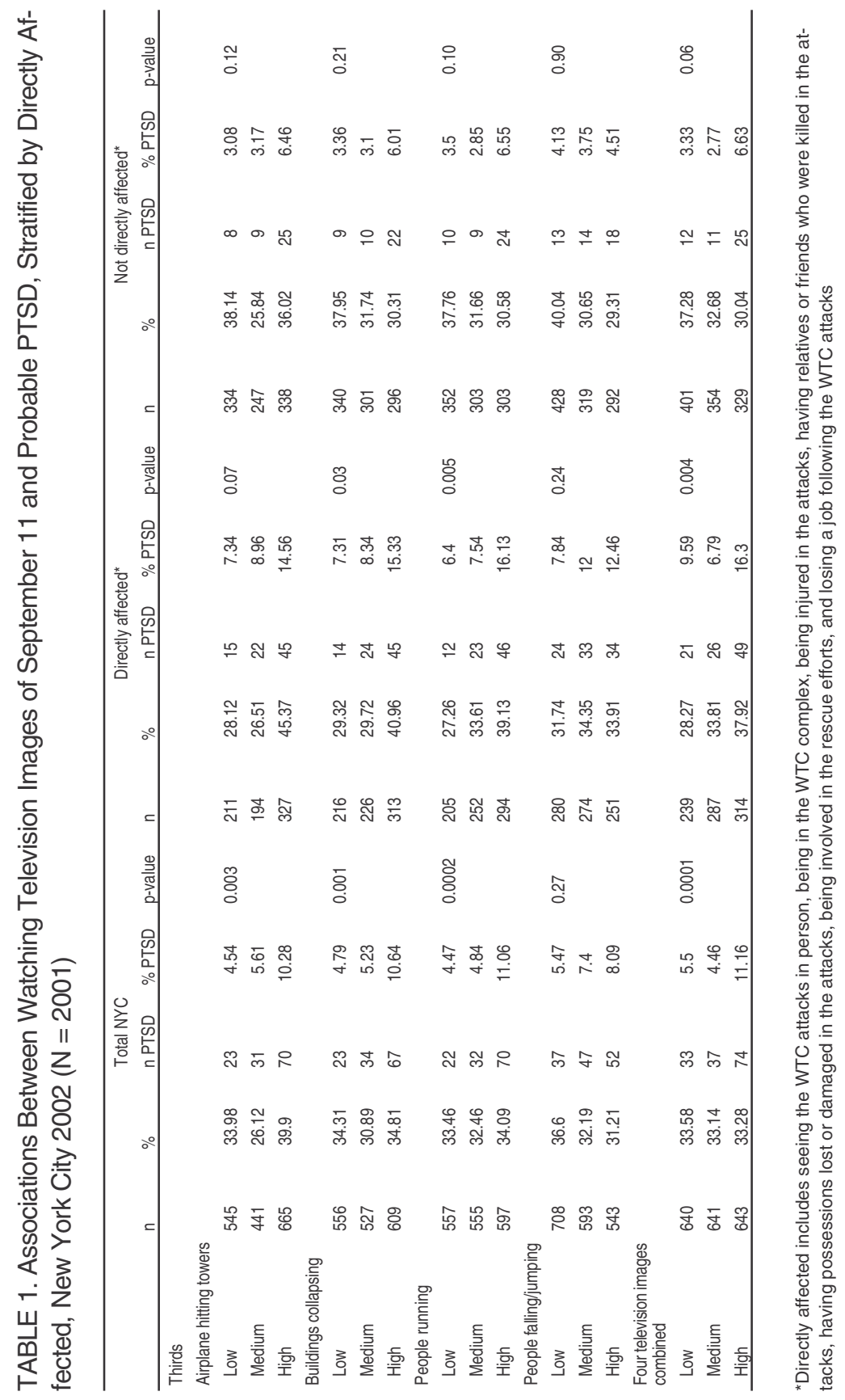


ning, they were $6.4 \%, 7.5 \%$, and $16.1 \%(p=0.005)$; and for the image of people falling or jumping, they were $7.8 \%, 12.0 \%$, and $12.5 \%(p=0.24)$. For the sum of all images combined, the prevalence of probable PTSD was 9.6\%, $6.8 \%$, and $16.3 \%$ ( $p=0.004)$ for low, medium, and high thirds of watching, respectively.

Among those not directly affected by the attacks, there were no significant associations between television images viewed and probable PTSD, although there was a borderline association with the sum of all images. The prevalence of probable PTSD in the highest category of watching combined images was $6.6 \%$, while it was $2.8 \%$ and $3.3 \%$ in the medium and low categories, respectively ( $p=0.06$; see Table 1$)$.

Examining the association between television watching and probable PTSD in combined groups of directly affected and past traumatic experiences revealed that associations were not consistent across strata (see Table 2). Among those who were both directly affected and experienced a prior potentially traumatic event, there was an association between television watching and probable PTSD: the prevalences for increasing thirds of watching were $12.1 \%, 7.9 \%$, and $15.6 \%(p=0.05)$. There were also associations between television watching and probable PTSD in those directly affected but with no past trauma, and in those with past trauma who were not directly affected $(p=$ $0.02, p=0.01$, respectively). However, among those who were not directly affected and had no past trauma there was no association between television watching and probable PTSD; the prevalences from low to high frequency of watching were $3.6 \%, 1.9 \%$, and $0.9 \%(p=0.37)$.

By combined categories of directly affected and peri-event panic, the association between watching television images and probable PTSD was not consistent across strata (see Table 3). Among those who experienced peri-event panic and were directly affected, the prevalence of probable PTSD was high (20-40\%) but did not differ by frequency of watching television images $(p=$ 0.37). Among those who experienced peri-event panic but were not directly affected, the prevalence of probable PTSD was high as well, but with no relation to television watching $(p=0.29)$. Among those who did not experience peri-event panic, the directly affected and not directly affected groups both had borderline associations between television watching and probable PTSD ( $p=0.06, p=0.07$, respectively).

\section{DISCUSSION}

In this analysis, we showed an association between watching television related to September 11 and probable PTSD both for a range of different images 
TABLE 2. Association Between Television Images and Probable PTSD, Stratified by Directly Affected and Past Traumatic Experience, New York City $2002(\mathrm{~N}=2001)$

\begin{tabular}{|c|c|c|c|c|c|c|c|c|c|c|c|}
\hline & \multirow{2}{*}{$\begin{array}{l}\text { Four television } \\
\text { images } \\
\text { combined }\end{array}$} & \multicolumn{5}{|c|}{ Directly affected* } & \multicolumn{5}{|c|}{ Not directly affected* } \\
\hline & & $\mathrm{n}$ & $\%$ & n PTSD & $\%$ PTSD & $p$-value & $\mathrm{n}$ & $\%$ & $\mathrm{n}$ PTSD & $\%$ PTSD & $p$-value \\
\hline \multirow{3}{*}{$\begin{array}{l}\text { Past traumatic } \\
\text { events }\end{array}$} & Low & 167 & 25.83 & 17 & 12.14 & 0.05 & 241 & 33.73 & 9 & 3.16 & 0.01 \\
\hline & Medium & 228 & 34.77 & 25 & 7.87 & & 249 & 33.56 & 9 & 3.19 & \\
\hline & High & 247 & 39.39 & 40 & 15.61 & & 244 & 32.71 & 23 & 8.94 & \\
\hline \multirow{3}{*}{$\begin{array}{l}\text { No past traumatic } \\
\text { events }\end{array}$} & Low & 72 & 35.94 & 4 & 3.84 & 0.02 & 160 & 44.03 & 3 & 3.58 & 0.37 \\
\hline & Medium & 59 & 30.78 & 1 & 2.97 & & 105 & 31.01 & 2 & 1.92 & \\
\hline & High & 67 & 33.27 & 9 & 18.85 & & 85 & 24.96 & 2 & 0.89 & \\
\hline
\end{tabular}

* Directly affected includes seeing the WTC attacks in person, being in the WTC complex, being injured in the attacks, having relatives or friends who were killed in the attacks, having possessions lost or damaged in the attacks, being involved in the rescue efforts, and losing a job following the WTC attacks 
TABLE 3. Association Between Television Images and Probable PTSD, Stratified by Directly Affected and Peri-Event Panic, New York City $2002(\mathrm{~N}=2001)$

\begin{tabular}{|c|c|c|c|c|c|c|c|c|c|c|c|}
\hline & \multirow{2}{*}{$\begin{array}{l}\text { Four television } \\
\text { images combined }\end{array}$} & \multicolumn{5}{|c|}{ Directly affected ${ }^{*}$} & \multicolumn{5}{|c|}{ Not directly affected ${ }^{*}$} \\
\hline & & $\mathrm{n}$ & $\%$ & $\mathrm{nPTSD}$ & $\%$ PTSD & $p$-value & $\mathrm{n}$ & $\%$ & n PTSD & $\%$ PTSD & $p$-value \\
\hline \multirow[t]{3}{*}{ Peri-event panic } & Low & 33 & 20.72 & 11 & 37.23 & 0.37 & 43 & 29.97 & 9 & 20.56 & 0.29 \\
\hline & Medium & 46 & 27.75 & 13 & 23.12 & & 37 & 28.31 & 4 & 8.25 & \\
\hline & High & 88 & 51.54 & 31 & 35.03 & & 63 & 41.72 & 11 & 12.85 & \\
\hline No peri-event & Low & 206 & 30.28 & 10 & 4.57 & 0.06 & 358 & 38.42 & 3 & 1.23 & 0.07 \\
\hline \multirow[t]{2}{*}{ panic } & Medium & 241 & 35.42 & 13 & 3.39 & & 317 & 33.36 & 7 & 2.05 & \\
\hline & High & 226 & 34.3 & 18 & 8.82 & & 266 & 28.22 & 14 & 5.2 & \\
\hline
\end{tabular}

* Directly affected includes seeing the WTC attacks in person, being in the WTC complex, being injured in the attacks, having relatives or friends who were killed in the attacks, having possessions lost or damaged in the attacks, being involved in the rescue efforts, and losing a job following the WTC attacks 
and for a combined measure of frequency of television watching. The majority of this association was present among those directly affected by the September 11 attacks. Consistent with previous research (Ahern et al., 2002), these results suggest the importance of a personal connection (e.g., knowing someone who was killed, being in the WTC towers, being injured in the attacks) to the images watched. Those who have a personal connection to the images may be more traumatized by watching those images, or conversely, those with a personal connection who begin to develop psychopathology may be more inclined to watch television related to the precipitating incident.

When we examined the combined contribution of being directly affected by the attacks and having had prior traumatic experiences, we found that among those who were not directly affected by the attacks and who had experienced no prior traumatic events there was no relation between television watching and probable PTSD. However, among those who were directly affected by the attacks, who experienced a prior traumatic event, or both, watching television about the attacks was associated with symptoms of probable PTSD. If future research demonstrates that watching television images of a disaster increases the risk of posttraumatic stress symptoms among those directly affected by the attacks or with a prior traumatic experience, then a reduction in television viewing for this population would be an effective public health intervention to decrease post-disaster psychopathology. The relation between watching images of traumatic events and psychological symptoms among those with previous traumatic experiences has been demonstrated in experimental settings and also in relation to September 11 television watching (Kinzie et al., 1998, 2002); however, these findings have mainly been among those with preexisting PTSD or depression. Similarly, among the respondents to our survey who had posttraumatic stress symptoms as a result of prior traumatic experiences, television viewing related to September 11 may have reactivated symptoms. Our findings in a general population sample raise the question of whether prior traumatic experience in combination with current television watching of an unfolding disaster are sufficient to explain the observed association with probable PTSD, or whether pre-existing psychopathology related to a prior traumatic event is a necessary factor that may explain this relation. Research suggests that media may be a trigger of suppressed traumatic memories (Elliott, 1997) and thus may have the ability to affect those with prior traumatic experiences who have not developed PTSD. More research in this area would enrich our understanding of these associations.

Examining the relation between being directly affected by the September 11 attacks and peri-event panic, we found that among those who experienced peri-event panic, the prevalence of probable PTSD was very high but unrelated to the September 11 television images, regardless of whether respon- 
dents were directly affected. The strong association between panic and posttraumatic stress symptoms is consistent with research that demonstrates strong associations between panic and PTSD (Bryant \& Panasetis, 2001; Deering et al., 1996; Falsetti \& Resnick, 1997; Galea et al., 2002), but the association between television watching and panic has not been much explored (Ahern et al., in press). Among those who did not experience peri-event panic, there were borderline associations between television watching and probable PTSD for the directly affected and those not directly affected. In particular, the relation for those who were directly affected but did not panic was much reduced from the overall association between television watching and probable PTSD among the directly affected. These findings suggest the importance of experiencing peri-event panic reactions as a key part of the relation between television watching and posttraumatic stress symptoms.

There are some limitations that should be considered when interpreting this analysis. The cooperation rate of $65.3 \%$ suggests we were unable to interview a substantial proportion of the households we attempted to contact. However, the demographic similarity between the achieved sample and Census figures suggests our sample is representative. The associations observed between television and probable PTSD do not demonstrate causality. Extensive television viewing may be a marker of early psychopathology, or the viewing may contribute to symptoms of psychopathology. Longitudinal assessment that determined the timing of the development of symptoms in relation to television viewing would help address the question of causality.

\section{Future Directions}

The findings in this analysis generate some questions to be addressed in future research. The relation between television watching and probable PTSD among those with prior traumatic experiences raises the question about what types of prior traumatic events may be more important in this context. Research among refugees suggests that images with similarity to the original traumatic event are more likely to elicit a response (Kinzie et al., 1998), and the recency of the prior traumatic experience and the proximity of the current traumatic event geographically and culturally to the individual (Kinzie et al., 2002) may be important predictors of the response to televised images of the current event. Exploration of these issues in general population samples may help us understand the relation between television and probable PTSD in a group with prior traumatic experiences who were only exposed to the current potentially traumatic event through television.

The finding that a large portion of the relation between television watching and probable PTSD can be removed by accounting for the experience of a 
peri-event panic attack raises important questions about the nature of the relation between television watching and peri-event panic. Primarily, it would be interesting to explore whether watching more television images of a disaster can contribute to precipitating a panic attack, or whether those who experience a peri-event panic attack later watch more television because of the panic attack. Experimental research among patients with PTSD suggests that watching television images of distressing events can cause physiological arousal, suggesting that television images might contribute to the development of a panic attack (Kinzie et al., 1998). This is a question that merits further exploration.

In the current era of constant television news coverage of events across the globe, the potential impact of watching disasters, wars, terrorism, and other traumatic events on television will only grow as a concern. A greater understanding of the mechanisms behind the observed associations between television watching and posttraumatic stress may help us understand and better prepare for the effects of future disasters.

\section{REFERENCES}

Ahern, J., Galea, S., Resnick, H., Kilpatrick, D., Bucuvalas, M., Gold, J. et al. (2002). Television images and psychological symptoms after the September 11 terrorist attacks. Psychiatry, 65(4), 289-300.

Ahern, J., Galea, S., Resnick, H., \& Vlahov, D. (in press). Television images and probable PTSD after September 11: The role of background characteristics, event exposures and peri-event panic. The Journal of Nervous and Mental Disease.

Bryant, R., \& Panasetis, P. (2001). Panic symptoms during trauma and acute stress disorder. Behavior Research and Therapy, 39(8), 961-966.

Centers for Disease Control and Prevention. (1989). Diagnostic Interview Schedule (DIS). Health Status of Vietnam Veterans. Supplement C: Medical and Psychological Procedure Manuals and Forms (pp. 405-499). Atlanta, GA: Author.

Deering, C. G., Glover, S. G., Ready, D., Eddleman, H. C., \& Alarcon, R. D. (1996). Unique patterns of comorbidity in posttraumatic stress disorder from different sources of trauma. Comprehensive Psychiatry, 37(5), 336-346.

Elliott, D. M. (1997). Traumatic events: Prevalence and delayed recall in the general population. Journal of Consulting and Clinical Psychology, 65(5), 811-820.

Falsetti, S. A., \& Resnick, H. (1997). Frequency and severity of panic attack symptoms in a treatment seeking sample of trauma victims. Journal of Traumatic Stress, 10(4), 683-689.

Galea, S., Ahern, J., Resnick, H., Kilpatrick, D., Bucuvalas, M., Gold, J. et al. (2002). Psychological sequelae of the September 11 terrorist attacks in Manhattan, New York City. The New England Journal of Medicine, 346(13), 982-987.

Holloway, H. C., Norwood, A. E., Fullerton, C. S., Engel, C. C., \& Ursano, R. J. (1997). The threat of biological weapons, prophylaxis and mitigation of psychological and 
social consequences. The Journal of the American Medical Association, 278(5), 425-427.

Kilpatrick, D., Resnick, H., Freedy, J., Pelcovitz, D., Resnick, P., Roth, S. et al. (1998). The posttraumatic stress disorder field trial: Evaluation of the PTSD construct-Criteria A through E. In T. A. Widiger, A. J. Frances, H. A. Pincus, R. Ross, M. B. First, W. Davis, \& M. Kline (Eds.), DSM-IV Sourcebook (Vol. 4, pp. 803-844). Washington DC: American Psychiatric Association Press.

Kinzie, J. D., Boehnlein, J., Riley, C., \& Sparr, L. (2002). The effects of September 11 on traumatized refugees: Reactivation of posttraumatic stress disorder. The Journal of Nervous and Mental Disease, 190(7), 437-441.

Kinzie, J. D., Denney, D., Riley, C., Boehnlein, J., McFarland, B., \& Leung, P. (1998). A cross-cultural study of reactivation of posttraumatic stress disorder symptoms. The Journal of Nervous and Mental Disease, 186, 670-676.

Long, N., Chamberlain, K., \& Vincent, C. (1994). Effect of the Gulf War on reactivation of adverse combat-related memories in Vietnam veterans. Journal of Clinical Psychology, 50(2), 138-144.

Moyers, F. (1996). Oklahoma City bombing: Exacerbation of symptoms in veterans with PTSD. Archives of Psychiatric Nursing, 10(1), 55-59.

Nader, K. O., Pynoos, R. S., Fairbanks, L. A., Al-Ajeel, M., \& Al-Asfour, A. (1993). A preliminary study of PTSD and grief among the children of Kuwait following the gulf crisis. British Journal of Clinical Psychology, 32, 407-416.

Pfefferbaum, B. (2001). The impact of the Oklahoma City bombing on children in the community. Military Medicine, 166(suppl. 2), 49-50.

Pfefferbaum, B., Gurwitch, R. H., McDonald, N. B., Leftwich, M. J. T., Sconzo, G. M., Messenbaugh, A. K. et al. (2000). Posttraumatic stress among young children after the death of a friend or acquaintance in a terrorist bombing. Psychiatric Services, 52(3), 386-388.

Pfefferbaum, B., Moore, V. L., McDonald, N. B., Maynard, B. T., Gurwitch, R. H., \& Nixon, S. J. (1999). The role of exposure in posttraumatic stress in youths following the 1995 bombing. Journal of the Oklahoma State Medical Association, 92(4), 164-167.

Pfefferbaum, B., Nixon, S. J., Krug, R. S., Tivis, R. D., Moore, V. L., Brown, J. M. et al. (1999). Clinical needs assessment of middle and high school students following the 1995 Oklahoma City bombing. American Journal of Psychiatry, 156(7), 1069-1074.

Pfefferbaum, B., Nixon, S. J., Tivis, R. D., Doughty, D. E., Pynoos, R. S., Gurwitch, R. H. et al. (2001). Television exposure in children after a terrorist incident. Psychiatry, 64(3), 202-211.

Pfefferbaum, B., Nixon, S. J., Tucker, P. M., Tivis, R. D., Moore, V. L., Gurwitch, R. H. et al. (1999). Posttraumatic stress responses in bereaved children after the Oklahoma City bombing. Journal of the American Academy of Child and Adolescent Psychiatry, 38(11), 1372-1379.

Pfefferbaum, B., Seale, T. W., McDonald, N. B., Brandt, E. N., Rainwater, S. M., Maynard, B. T. et al. (2000). Posttraumatic stress two years after the Oklahoma City bombing in youths geographically distant from the explosion. Psychiatry, 63(4), 358-370. 
Pittman, R. K., Orr, S. P., Forgue, D. F., deJong, J., \& Claiborn, J. M. (1987). Psychophysiologic assessment of posttraumatic stress disorder imagery in Vietnam combat veterans. Archives of General Psychiatry, 44, 970-975.

Resnick, H. S., Kilpatrick, D. G., Dansky, B. S., Saunders, B. E., \& Best, C. (1993). Prevalence of civilian trauma and posttraumatic stress disorder in a representative national survey of women. Journal of Consulting and Clinical Psychology, 61, 984-991.

Schlenger, W. E., Caddell, J. M., Ebert, L., Jordan, B. K., Rourke, K. M., Wilson, D. et al. (2002). Psychological reactions to terrorist attacks. Findings from the national study of Americans' reactions to September 11. The Journal of the American Medical Association, 288(5), 581-588.

Schuster, M. A., Stein, B. D., Jaycox, L. H., Collins, R. L., Marshall, G. N., Elliott, M. N. et al. (2001). A national survey of stress reactions after the September 11, 2001, terrorist attacks. The New England Journal of Medicine, 345(20), 1507-1512.

Shah, B., Barnwell, B., \& Bieler, G. (1997). SUDAAN user's manual, release 7.5. Research Triangle Park, NC: Research Triangle Institute.

Silver, R. C., Holman, E. A., McIntosh, D. N., Poulin, M., \& Gil-Rivas, V. (2002). Nationwide longitudinal study of psychological responses to September 11. The Journal of the American Medical Association, 288(10), 1235-1244.

Spitzer, R., Williams, J., Gibbon, M., \& First, M. (1992). The structural clinical interview for DSM-III-R (SCID) I: History, rationale, and description. Archives of General Psychiatry, 49, 624-629. 